\title{
High-intensity focused ultrasound therapy: effects on urinary and erectile function and quality of life
}

Several minimally invasive treatments for patients with localized prostate cancer have been developed with the aim of avoiding the risks associated with open surgery. High-intensity focused ultrasound (HIFU) therapy employs a targeted ultrasound beam that causes heatinduced coagulative necrosis of the tumor without affecting the surrounding healthy tissue and while minimizing the risk of damage to the neurovascular bundles. HIFU has been shown to be oncologically effective, but its effects on quality of life (QOL), particularly relating to urinary and erectile function, are also important to patients who are considering their treatment options. Results of a Japanese

\section{4 ...HIFU therapy seems to} be comparable or superior to other minimally invasive treatments... 77 prospective study suggest that the QOL outcomes following HIFU therapy in patients with localized prostate cancer are comparable to or better than those for other minimally invasive modalities.

Between 1999 and 2007, 558 men underwent HIFU therapy at Tokai University Hachioji Hospital. Patients who received neoadjuvant hormone deprivation therapy were excluded from the final analysis, resulting in a study population of 326 eligible patients. The mean age of the participants was 68 years (range 45-88 years), and the mean prostate volume was $21.7 \mathrm{ml}$ (range $7.1-45.8 \mathrm{ml}$ ). The 18 patients with prostate volume $>40 \mathrm{ml}$ underwent transurethral resection of the prostate 1 month before HIFU.

At 6, 12 and 24 months after HIFU therapy, mean International Prostate Symptom Score (IPSS) and International Index of Erectile Function 5 (IIEF-5) score were not different from baseline values. Mean maximum urinary flow rate significantly decreased and postvoid residual urine volume significantly increased at 6 months, but returned to baseline levels at 12 and 24 months. General health-related QOL had improved markedly after 24 months, and prostatespecific QOL did not change significantly at any time point. Overall potency rates at 6,12 and 24 months were $52 \%, 63 \%$ and $79 \%$, respectively.

The authors conclude that HIFU therapy seems to be comparable or superior to other minimally invasive treatments in patients with localized prostate cancer in terms of urinary and erectile function.

\section{Nick Warde}

Original article Shoji, S. et al. Quality of life following high-intensity focused ultrasound for the treatment of localized prostate cancer: a prospective study. Int. J. Urol. 17, 715-719 (2010) 